\section{§13. Inter-machine Validation Study of Neoclassical Transport Modelling in Medium- to High-density Stellarator-Heliotron Plasmas}

Dinklage, A. ${ }^{1}$, Yokoyama, M. ${ }^{2}$, Tanaka, K. ${ }^{2}$, Velasco, J.L. ${ }^{3}$, López-Bruna, D. ${ }^{3}$, Beidler, C.D. ${ }^{1}$, Satake, S. ${ }^{2}$, Ascasibar, E. ${ }^{3}$, Arevalo, J. ${ }^{3}$, Baldzuhn, J. ${ }^{1}$, Feng, Y. ${ }^{1}$, Gates, D. ${ }^{4}$, Geiger, J. ${ }^{1}$, Ida, K. ${ }^{2}$, Isaev, M. ${ }^{5}$, Jakubowski, M. ${ }^{1}$, López-Fraguas, A. ${ }^{3}$, Maaßberg, H. ${ }^{1}$, Miyazawa, J. ${ }^{2}$, Morisaki, T. ${ }^{2}$, Murakami, S. ${ }^{6}$, Pablant, N. ${ }^{4}$, Kobayashi, S. ${ }^{2}$, Seki, R. ${ }^{2}$, Suzuki, C. ${ }^{2}$, Suzuki, Y. ${ }^{2}$, Turkin, Y. ${ }^{1}$, Wakasa, A. ${ }^{6}$, Wolf, R. ${ }^{1}$, Yamada, H. ${ }^{2}$, Yoshinuma, M. ${ }^{2}$, LHD Experiment Group ${ }^{2}$, TJ-II Team ${ }^{3}$, W7-AS Team ${ }^{1}$ ${ }^{1}$ Max-Planck-Institut für Plasmaphysik, EURATOM Association, Greifswald, Germany

${ }^{2}$ National Institute for Fusion Science, Toki, Japan

${ }^{3}$ CIEMAT, EURATOM Association, Madrid, Spain

${ }^{4}$ Princeton Plasma Physics Laboratory, Princeton, NJ, USA

${ }^{5}$ National Res. Cent. 'Kurchatov Institute', Moscow, Russia

${ }^{6}$ Dep. Nuclear Engineering, Kyoto Univ., Kyoto, Japan

An inter-machine dataset covering devices of different size and a variety of magnetic configurations is comprehensively analysed to assess the ranges of validity of neoclassical (NC) transport predictions in medium- to high density, high temperature discharges. A recently concluded benchmarking of calculations of transport coefficients from local NC theory [1] allows now a quantitative experimental energy transport study. While in earlier inter-machine studies of NC transport in 3D devices the electron energy transport at low densities has been investigated [2], this study focuses on the energy transport at medium to higher densities as anticipated when approaching reactor conditions. The validation approach as done here is to compare two fluxes: first, the 'NC flux' is determined with the $\mathrm{NC}$ transport coefficients and the gradients of the experimental density and temperature profiles. Second, the sources from deposition calculations considering heating and particle sources (the latter where available) yield the 'experimental flux'. Both fluxes are compared and the $\mathrm{NC}$ radial electric field $E^{\mathrm{NC}}{ }_{r}$ was compared with measurements of $E_{r}$ where available. Technically, the steady-state energy balance analyses were performed using the integrated transport code, TASK3D [3] for LHD discharges. Fig. 1 shows an example for an energy transport analysis for LHD. More details are to be found in Ref. [4].

In TJ-II the NC particle fluxes and electron energy fluxes agree within $r / a<1 / 2 \sim 2 / 3$. The NC prediction for the ion energy flux does not match at all. The radial electric field is found to reflect ion-root conditions but differs from measurements significantly $\left(\left|E^{\mathrm{NC}_{r}}\right|<\left|E^{\exp _{r}}\right|\right)$. For the assessed W7-AS plasmas, $\mathrm{NC}$ theory is consistent with experimental findings for $F$ and $Q_{\text {e. }}$ within $r / a<2 / 3$. There are some differences in $E_{r}$ for the outermost radii. In the selected LHD discharges, large contributions of NC ion transport to the overall energy fluxes are found both in the standard and the inward-shifted magnetic configuration for $r / a<0.7$. The electron energy flux complies with $\mathrm{NC}$ predictions for the $\mathrm{STD}(\mathrm{R}=3.75 \mathrm{~m})$ case in the same region but appears to be underpredicted for the IWD $(\mathrm{R}=3.6 \mathrm{~m})$ configuration even in the core region. The radial electric field measured by CXRS shows differences to the ambipolar $E_{r}$ specifically for the STD case in the plasma core.
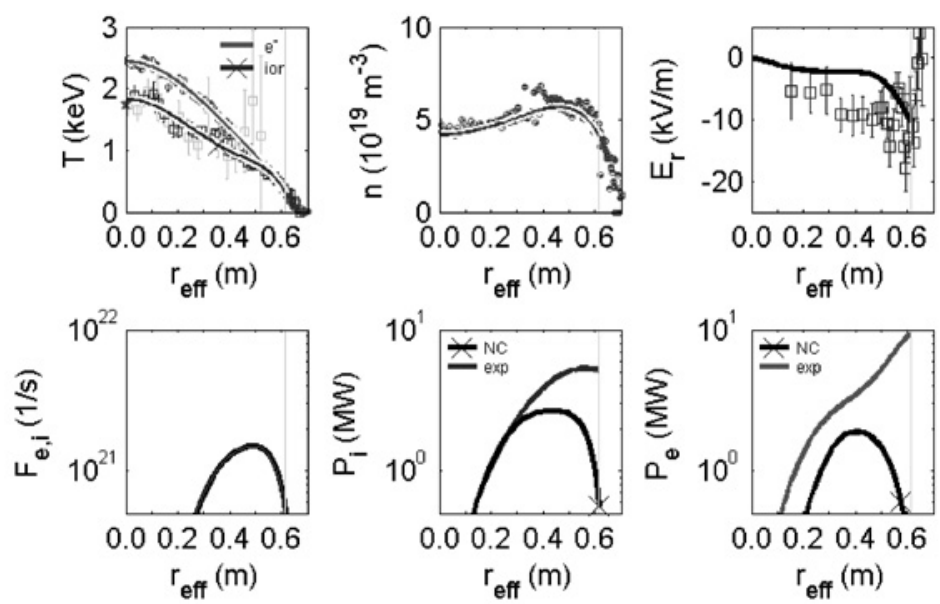

Fig. 1. Transport analysis for LHD discharge \#109696 $(\mathrm{t}=4.44 \mathrm{~s}) . T_{\mathrm{e}}$ and $n_{\mathrm{e}}$ comes from Thomson scattering, $T_{\mathrm{i}}$ from CXRS (grey $T_{\mathrm{i}}$ data from inboard-side measurements) and a central value from X-ray imaging (magenta star). $E_{r}$ data are $\delta t \pm 15 \mathrm{~ms}$ weighted-averaged CXRS measurements. $\mathrm{F}$ are the particle fluxes and $\mathrm{P}$ are the energy fluxes (lower row) for experimental (exp.) and neoclassical (NC).

For densities $n>4 \times 10^{19} \mathrm{~m}^{-3}$ and temperatures above some $\mathrm{keV}$, it can be concluded that the NC energy fluxes significantly contribute to or even fully comply with experimental findings in the plasma core region. Nevertheless, larger discrepancies to NC predictions are found in adjacent parameter regimes in LHD (e.g. [5,6]). For the peripheral region, the energy transport cannot be explained by $\mathrm{NC}$ transport. In line with this result, fluctuation measurements with phase-contrast imaging indicate fluctuation amplitudes being maximum in the periphery at wavenumbers compliant with ITG or TEM turbulence. The global confinement for the assessed parameter range complies well with the ISS04, gyro-Bohm type energy confinement scaling for LHD and W7-AS.

The joint experiment conducted in LHD was supported by National Institute for Fusion Science (NIFS) /National Institutes of Natural Sciences (NINS) under the project, 'Promotion of the International Collaborative Research Network Formation' (NIFSKEIN1107). This work is also partly supported under the auspices of the NIFS Collaboration Research program (NIFSKNTT008 and NIFSKLPT001).

1)Beidler, C.D. et al. Nucl. Fusion 51 (2011) 076001

2)Yokoyama, M. et al. Nucl. Fusion 47 (2007) 1213

3)Yokoyama, M. et al. Plasma Fusion Res. 7 (2012) 2403011

4) Dinklage, A. et al. Nucl. Fusion (at press)

5) Tanaka, K. et al.: Plasma Fusion Res. 5 (2010) S2053.

6) Ida, K. et al. Phys. Rev. Lett. 91 (2003) 085003 